\title{
Estudios de Validez del Youth Self Report/11-18 en Adolescentes Mexicanos
}

\author{
Validity Studies of Youth Self Report/11-18 in Mexican Adolescents
}

\author{
Blanca Estela Barcelata-Eguiarte ${ }^{1}$ y María Elena Márquez-Caraveo ${ }^{2}$
}

\begin{abstract}
Resumen
El objetivo de este estudio fue evaluar la validez de constructo y consistencia interna de la versión oficial internacional del ASEBA del YSR/11-18 en español en adolescentes mexicanos. Se seleccionó de manera intencional una muestra de 790 estudiantes de 13 a 18 años de secundarias y bachilleratos públicos de la zona metropolitana de la Ciudad de México. La muestra se dividió en dos partes o muestras; con la primera se realizó un análisis factorial exploratorio y con la segunda se llevó a cabo un análisis factorial confirmatorio. Se encontró una estructura de doce factores de Banda Estrecha, ocho originales y cuatro nuevos factores, que se agruparon en cuatro factores de Banda Ancha, Internalizados, Externalizados y Mixtos, y uno nuevo de Cualidades Positivas como se reporta en investigación reciente. La estructura factorial en este estudio, presenta adecuadas propiedades psicométricas por lo cual podría ser útil para diferenciar problemas de salud mental como recursos en los adolescentes.
\end{abstract}

Palabras clave: $Y S R / 11-18$, validez de constructo, confiabilidad, análisis factorial confirmatorio, adaptación adolescente

\begin{abstract}
The objective of this study was to evaluate the construct validity and the reliability of the official international Hispanic version of the YSR/11-18 in Mexican adolescents. A sample of 790 students aged 13 to 18 years was intentionally recruited from public secondary and high schools in the metropolitan zone of Mexico City. This sample was divided in two parts or samples. An exploratory factor analysis was carried out with sample one and a confirmatory factor analysis was conducted with data from sample two. A structure of twelve Narrow Band factors was observed, eight original factors and four new factors. These were integrated in four Broad Band factors: Internalizing, Externalizing, and Mixed, and a new one of Positive Qualities, as reported in recent research. The factorial structure in this study presents adequate psychometric properties and seems to be useful both to evaluate mental health problems and adolescents' resources.
\end{abstract}

Keywords: $Y S R / 11-18$, construct validity, reliability, confirmatory factor analysis, adolescent adaptation

\footnotetext{
${ }^{1}$ Doctora en Psicología y Salud. Profesor de Tiempo Completo Titular B, Universidad Nacional Autónoma de México. Prol. Abasolo 307-7, Col. Valle de Tepepan, Tlalpan, CdMx. CP: 14646, México. Tel.: +5255-56230597. Correo: bareg7@ hotmail.com

${ }^{2}$ Doctora en Psicología y Salud. Jefa de la División de Investigación, Hospital Psiquiátrico Infantil Dr. Juan N. Navarro. San Buenaventura 86, Col. Belisario Domínguez, Tlalpan, CdMx. CP: 14080, México. Tel.: +5255-63888026. Correo: malenamarquezc@gmail.com
} 


\section{Introducción}

Uno de los objetivos del enfoque ecológicotransaccional del desarrollo, es predecir las trayectorias del desarrollo de los adolescentes que dan por resultado desenlaces positivos o negativos (Cicchetti \& Rogosch, 2002). La medición de estos resultados es esencial por su implicación para la práctica clínica y para la comparación de datos de adolescentes de diversos contextos (Deighton et al., 2014). Disponer de herramientas válidas y confiables permite la identificación temprana de trastornos, lo que contribuye a la planeación de acciones de salud mental, con instrumentos científicamente probados. De ahí la importancia de evaluar las propiedades psicométricas y la pertinencia de los instrumentos que evalúan la salud mental de los adolescentes en distintos grupos poblacionales (Achenbach, Rescorla, \& Ivanova, 2012).

El enfoque taxonómico de los trastornos de salud mental (p. e. CIE-10, DSM5), tiene respaldo teórico y clínico (Lacalle, 2009), pero identifica muchos falsos positivos y muestra un exceso de comorbilidad a nivel diagnóstico (Widakowich, 2012). Por el contrario, el enfoque dimensional asume que los comportamientos de niños y adolescentes pueden variar en grado como parte de un continuo y ser condensados en grupos de síntomas o problemas (Achenbach \& Rescorla, 2001). Estos problemas se cuantifican y clasifican con base empírica por medio de procedimientos estadísticos multivariados que hacen potencialmente más objetiva y fiable la evaluación (Lacalle, 2009) adhiriéndose a una visión "transdiagnóstica" (Achenbach, 2015).

Una de las medidas dimensionales de mayor uso para la evaluación de problemas emocionales, de conducta y del funcionamiento adaptativo en diferentes países es el Youth Self Report para adolescentes de 11 a 18 años (YSR/11-18) el cual forma parte del Sistema de Evaluación Empírica (Achenbach System of Empirically Based Assessment, ASEBA, por sus siglas en inglés) propuesto por Achenbach y Rescorla (2001). El $Y S R / 11-18$, es un auto-reporte que evalúa el comportamiento, el funcionamiento y la adaptación adolescente. La estructura original presenta dos partes: la primera valora competencias psicosociales, recreativas, deportivas y académicas; la segunda, identifica la presencia de problemas y de conductas adaptativas, a través de 112 ítems en una escala Likert $(0=\mathrm{No}$ es cierto, $1=\mathrm{En}$ cierta manera, algunas veces, $3=$ Muy cierto 0 cierto a menudo). Los problemas están integrados en ocho factores de primer orden denominados Banda Estrecha (BE): $\quad 1 . \quad$ Ansiedad/Depresión, 2. Retraimiento/Depresión, 3. Quejas Somáticas, 4. Ruptura de Reglas, 5. Conducta Agresiva, 6. Problemas Sociales, 7. Problemas de Pensamiento, y 8. Problemas de Atención. Estos factores, a su vez, se agrupan en factores de segundo orden o de Banda Ancha (BA). Los tres primeros corresponden a los Problemas Internalizados (PI), los dos siguientes son conocidos como Problemas Externalizados (PE) y los tres factores restantes se identifican como Problemas Mixtos (PM) porque no se agrupan ni en los Problemas Internalizados ni en los Externalizados (Achenbach \& Rescorla, 2001).

Los Problemas Internalizados implican un elevado control y aluden a condiciones cuya característica principal es la emoción o el estado de ánimo alterado; mientras que los Problemas Externalizados reflejan un bajo control y engloban a todos aquellos comportamientos observables que reflejan conflictos de adaptación social y que involucran a otras personas, como mentir, robar, agredir o presentar conductas delictivas (Achenbach \& Rescorla, 2001; Valencia \& Andrade, 2005). Los Problemas Mixtos se relacionan con otro tipo de alteraciones tales como los problemas de pensamiento o los problemas de atención. Por otra parte, existen algunos comportamientos evaluados por el YSR/11-18 que implican aspectos positivos de los adolescentes y que son agrupados más recientemente en un factor de Banda Ancha que implica características positivas (Daset, López, \& Hidalgo, 2009; Ivanova et al., 2007).

El YSR/11-18 ha sido traducido a más de 80 idiomas (Deighton et al., 2014; Verhulst \& Achenbach, 1995) utilizado ampliamente en diversos países constituyéndose en una herramienta diagnóstica y de tamizaje de problemas de salud mental para adolescentes por lo que cuenta con varios estudios sobre sus propiedades psicométricas bajo el supuesto de que los comportamientos varían en función del 
género, la edad y la cultura. La estructura presentada por Achenbach y Rescorla (2001) ha sido confirmada principalmente en Estados Unidos de América así como en otras sociedades a través de estudios comparativos (p. e. Ivanova et al., 2007; Rescorla et al., 2007). Un estudio transcultural de Rescorla y colaboradores (2007) con muestras de 24 países corroboró los dos factores de segundo orden correspondientes a Problemas Internalizados y Externalizados así como uno más de Conductas Positivas, aunque fue menos consistente. Las correlaciones oscilaron de .55 a .72 , mostrando alta consistencia interna a lo largo de los países $\left(\alpha_{\text {promedio }}=.94\right)$. Las chicas mostraron más Problemas Internalizados que los chicos, en quienes predominaron los Externalizados, aunque la cultura mostró un mayor tamaño del efecto que el sexo. Estos hallazgos sugieren que los factores de segundo orden o de Banda Ancha son más estables, como lo demuestran varios estudios (Ivanova et al., 2007; Rescorla et al., 2007; 2012); mientras que los de primer orden o de Banda Estrecha tienden a variar más, dependiendo de la cultura. Esto se observa, particularmente, en los estudios realizados con población de habla hispana.

Gran parte de la evidencia del YSR/11-18 con población de habla hispana se encuentra en España. Los distintos autores reportaron variaciones en su estructura general, de acuerdo a las muestras de las diferentes regiones. En Asturias, Lemos, Vallejo y Sandoval (2002) confirmaron tres factores de segundo orden o de Banda Ancha (Internalizados, Externalizados y Mixtos), También reportaron nueve factores de primer orden o de Banda Estrecha (Varianza Explicada 70\%), tanto para chicas como para chicos de una muestra comunitaria de adolescentes. Estos factores resultaron de la depuración de 15 factores iniciales. Depresión y Ansiedad se agruparon por separado a diferencia de la versión de Achenbach y Rescorla (2001) y surgió un nuevo factor de Trastornos de Conducta. Aunque los estudios previos (Lemos, Fidalgo, Calvo, \& Menéndez, 1992a, 1992b) no reportan validez de constructo, hacen referencia a conductas socialmente deseables. Sandoval, Lemos y Vallejo (2006), en una investigación posterior, compararon jóvenes asturianos y madrileños, con el fin de verificar si había diferencias debidas al contexto. Mostraron una estructura de BA compuesta por Problemas Internalizados y Externalizados y ocho de BE, encontrando además diferencias por sexo, aunque igual número de factores, siendo las chicas quienes reportaron más problemas, en particular Problemas Internalizados, que correlacionaron de manera negativa con las competencias sociales (Lemos et al., 1992a; 2002). En Cataluña, Abad, Forns, Amador y Martorell (2000) reportaron una estructura de nueve factores en chicos y siete factores en chicas, con alfas de .56 a .74 . Se observaron puntajes mayores en Problemas Internalizados en las chicas y en los chicos más Problemas Externalizados. Otro estudio realizado en Granada para evaluar competencias sociales también se basó en una estructura de siete factores (Zubeidat, Fernández, Ortega, Vallejo, \& Sierra, 2009) encontrándose mayores Problemas Externalizados en los chicos y más Problemas Internalizados en las chicas. Estos estudios concuerdan en que las chicas tienden a presentar mayor número de Problemas Internalizados y los chicos más Externalizados, aunque éstos, también presentaron más competencias sociales.

En Latinoamérica, el estudio de Bordin y colaboradores (2013) con adolescentes brasileños confirmó la estructura original de Banda Ancha y Banda Estrecha reportada por Achenbach y Rescorla (2001). Por su parte, Daset y colaboradores (2009) observaron una agrupación de cinco nuevos síndromes en un grupo de adolescentes uruguayos: Conducta Disocial, Inmadurez, Temor, Problemas Sociales y uno denominado Síndrome Prosocial, que es el equivalente al de Cualidades Positivas, reportado previamente (Ivanova et al., 2007; Lemos et al., 1992a). En Perú, Alarcón y Bárrig (2015), evaluaron a chicos $y$ chicas adolescentes utilizando la versión de ocho factores $(\alpha=.93)$ y confirmaron las diferencias de género reportadas en la literatura internacional que indica que los chicos presentan más problemas Externalizados, en particular en Ruptura de Reglas y en Conducta Agresiva, en comparación con las chicas, quienes puntuaron más alto en el total de Problemas Internalizados, específicamente en los de Ansiedad, Depresión y Quejas Somáticas, así como en Problemas de Atención, aunque no se reportan datos de validez de constructo. El estudio 
de Ivanova y colaboradores (2007) para la generalización de la taxonomía de los problemas del YSR/11-18, incluyó muestras de 23 países, entre ellos Jamaica y Puerto Rico, y confirmó la estructura factorial reportada por Achenbach y Rescorla (2001) con indicadores de ajuste aceptables para estos dos países (p.e. $\mathrm{CFI}=.838$ y $\mathrm{CFI}=.900$, respectivamente).

En México, estudios previos (Betancourt, 2007; Valencia \& Andrade, 2005), exploraron la replicabilidad de la estructura original del YSR/1118 propuesta por Achenbach y Rescorla (2001) en diferentes rangos de edad. El estudio de Valencia y Andrade (2005), realizado con escolares de 9 a 15 años, reportó una versión modificada con ítems con cuatro opciones de respuesta ( 0 al 3 ) diferente a la versión de Achenbach y Rescorla (2001). Sus datos se agruparon en seis factores: Problemas Externalizados, Depresión/Ansiedad, Problemas Somáticos, Problemas de Pensamiento, Problemas Afectivos, y Problemas de Ansiedad, dos menos que la original ( $\mathrm{VE}=30.7 \%)$. Un estudio posterior (Betancourt, 2007) utilizó la versión de Valencia y Andrade (2005) que fue modificada nuevamente para población adolescente $\left(M_{\text {edad }}=14\right.$ años), dando como resultado una configuración inicial de 32 factores, ajustada a 11 factores $(\mathrm{VE}=31.8 \%$ ), incluidos cuatro factores nuevos no reportados previamente: Consumo de Alcohol y Tabaco, Conducta Desafiante, Problemas Interpersonales y Conductas Autoinfligidas. Estos estudios reportaron cambios sustanciales de la versión en inglés del YSR/11-18 (Achenbach \& Rescorla, 2001), con una opción más de respuesta y estructuras factoriales diferentes dando como resultado un nuevo instrumento denominado "Escala de Problemas Emocionales y de Conducta" de Betancourt y Andrade (2010) que difiere del YSR/11-18, aunque evalúa aspectos similares como Depresión, Rompimiento de Reglas, Consumo de Alcohol, Problemas Somáticos, Conducta Agresiva, Problemas de Pensamiento y Lesiones Autoinfligidas (Andrade, Betancourt, \& Vallejo, 2010). También encontraron que las chicas presentan más Problemas Internalizados y los chicos más Externalizados. En este sentido, se observa que no se reportan datos de validación de la versión oficial internacional en español del YSR/11-18 del $A S E B A$, con adolescentes mexicanos conocida también como "Cuestionario sobre el comportamiento de niños(as) de 11 a 18 años", como tampoco se reportan análisis factoriales confirmatorios en dicha población. Esto refleja algunas limitaciones para el uso confiable del YSR/11-18 en adolescentes mexicanos que facilite la investigación transcultural.

En suma, existen variaciones en la estructura factorial del $Y S R / 11-18$ en los diversos grupos, contextos y países, incluido México. También se reportan diferencias debidas al sexo. Aunque las chicas tienden a presentar más Problemas Internalizados y los chicos más Problemas Externalizados, los resultados varían en los factores de BE. Los estudios preliminares con adolescentes mexicanos, no utilizaron la versión oficial internacional en español y derivaron en un instrumento, que aunque también evalúa problemas de conducta, es diferente al YSR/11-18. Por tanto, el objetivo de este estudio fue analizar la validez de constructo y la consistencia interna de la versión oficial internacional en español del Youth Self Report/11-18 (YSR/11-18) (Achenbach $\&$ Rescorla, 2001) que forma parte del ASEBA (Achenbach et al., 2012), en una muestra de adolescentes mexicanos. Lo anterior en virtud de que es fundamental contar con medidas válidas y confiables para población mexicana como el $Y S R / 11-18$, que ha mostrado ser útil en la identificación de problemas emocionales y de conducta en muchos países. Esto permitiría identificar oportunamente alteraciones en las trayectorias del desarrollo y diseñar intervenciones más precisas a partir de una perspectiva preventiva (Compas et al., 2015; Freire, Teixeira, Silva, \& Matias, 2014). Esto es particularmente necesario en países de ingreso medio y bajo como México, ya que los niños y adolescentes representan la franja más ancha de la pirámide poblacional y existe evidencia respecto a la escasez de intervenciones psicosociales con base empírica y enfoque cultural, a pesar de la necesidad de que el equipo de salud mental considere los datos de su propia población al diseñar planes de acción (Kieling et al., 2011).

\section{Método}

Se realizó una investigación de campo transversal, de tipo instrumental de dos muestras 
(Ato, López, \& Benavente, 2013) avalada por el Programa de Apoyo a Proyectos de Investigación e Innovación Tecnológica (IN305917).

\section{Participantes}

Se seleccionaron de manera no probabilística un total de 790 adolescentes, de 13 a 18 años de edad $\left(M_{\text {edad }}=15.38 ; D E=1.58\right)$ de secundarias y bachilleratos públicos de las zonas norte, sur, oriente y poniente de la Ciudad de México. El método de selección tuvo el propósito de obtener una muestra equilibrada en función del sexo, la edad y la escolaridad en la que hubiera representación de adolescentes de diferentes zonas de la Ciudad dada la diversidad. Se incluyeron en la muestra estudiantes regulares, sin problemas de conducta, y que contaran con consentimiento y asentimiento informado. Con el fin de realizar estudios de validez paralelos a través de un análisis factorial exploratorio (AFE) y un análisis factorial confirmatorio (AFC) la muestra total se dividió de manera aleatoria en dos partes iguales (Tabla 1). La primera parte (muestra 1) estuvo compuesta por adolescentes con una edad promedio de $15.16(D E=1.52)$ y la segunda parte (muestra 2) estuvo integrada por adolescentes con edad promedio de $15.25(D E=1.58)$. Bajo el supuesto de que el AFE y AFC son dos métodos diferentes que forman parte de un continuo y de que el primero tiene el objetivo de explorar la estructura subyacente y el segundo probar el ajuste del modelo y su estabilidad, se consideró pertinente realizar los análisis con dos muestras o "submuestras" obtenidas de una muestra total (Ferrando \& Anguiano, 2010; Lloret, Ferreres, Hernández, \& Tomás, 2014).

\section{Instrumentos}

Se utilizó la versión oficial internacional en español del Youth Self Report/11-18 (YSR/11-18), del ASEBA, de Achenbach y Rescorla (2001). Esta versión se compone de dos partes: la primera valora competencias deportivas, sociales, recreativas y académicas, y la segunda evalúan problemas y conductas adaptativas a través de 112 ítems Likert de tres puntos $(0=$ no es cierto, $1=$ en cierta manera, algunas veces, $2=$ Muy cierto o cierto a menudo) que son los que se consideraron en este estudio. Como se mencionó anteriormente, es un instrumento que explora tres grandes grupos de problemas (Banda Ancha) que incluyen ocho tipos de problemas específicos (Banda Estrecha). El porcentaje de varianza explicada (VE) e indicadores de consistencia interna alfa $(\alpha)$ que se reportan son: Internalizados $(\mathrm{VE}=11 \% ; \alpha=.90): 1$. Ansiedad/Depresión $\quad(\mathrm{VE}=8 \% ; \quad \alpha=.84), \quad 2$. Retraimiento/Depresión $\quad(\mathrm{VE}=9 \% ; \quad \alpha=.80), 3$. Quejas Somáticas $\quad(\mathrm{VE}=8 \% ; \quad \alpha=.78)$; Externalizados (V.E=17\%; $\alpha=.94)$, 4. Ruptura de Reglas (VE=12\%; $\alpha=.85), 5$. Conducta Agresiva (VE $=16 \% ; \quad \alpha=.94), \quad$ y Mixtos: 6. Problemas Sociales (VE $=10 \% ; \alpha=.82)$, 7. Problemas de Pensamiento (VE=7\%; $\alpha=.78$ ), y 8. Problemas de Atención ( $\mathrm{VE}=9 \%, \alpha=.86)$. Adicionalmente, se aplicó la Cédula Sociodemográfica del Adolescente y su Familia (Barcelata, 2016) para caracterizar a los participantes, la cual consta de 16 ítems de opción múltiple que permiten obtener datos como el sexo, la edad, el estado civil, la escolaridad y la ocupación del adolescente y sus padres, así como el ingreso y la estructura familiar (alfa ordinal=.787).

Tabla 1. Características sociodemográficas de los participantes

\begin{tabular}{|c|c|c|c|c|c|c|}
\hline \multirow[t]{2}{*}{ Características } & \multicolumn{2}{|c|}{$\begin{array}{c}\text { Muestra } 1 \\
\mathrm{n}=395\end{array}$} & \multicolumn{2}{|c|}{$\begin{array}{c}\text { Muestra } 2 \\
n=395\end{array}$} & \multicolumn{2}{|c|}{$\begin{array}{c}\text { Muestra Total } \\
\mathrm{N}=790\end{array}$} \\
\hline & $\mathrm{n}$ & $\%$ & $\mathrm{n}$ & $\%$ & $\mathrm{~N}$ & $\%$ \\
\hline \multicolumn{7}{|l|}{ Sexo } \\
\hline Chicos & 188 & 47.59 & 199 & 50.49 & 387 & 48.95 \\
\hline Chicas & 207 & 52.40 & 196 & 49.6 & 403 & 51.01 \\
\hline \multicolumn{7}{|l|}{ Edad } \\
\hline 13 & 65 & 16.2 & 67 & 17.0 & 132 & 16.6 \\
\hline 14 & 72 & 18.4 & 69 & 17.5 & 141 & 17.9 \\
\hline 15 & 77 & 19.4 & 74 & 18.6 & 151 & 19.0 \\
\hline 16 & 66 & 16.6 & 69 & 17.5 & 135 & 17.1 \\
\hline 17 & 67 & 17.0 & 64 & 16.2 & 131 & 16.6 \\
\hline 18 & 48 & 12.4 & 52 & 13.2 & 100 & 12.8 \\
\hline \multicolumn{7}{|l|}{ Nivel escolar } \\
\hline Secundaria & 217 & 54.8 & 201 & 50.9 & 418 & 52.9 \\
\hline Bachillerato & 178 & 45.69 & 194 & 49.1 & 372 & 47.1 \\
\hline
\end{tabular}




\section{Procedimiento}

De acuerdo al objetivo de este estudio, se realizó una adaptación linguíística de la versión oficial internacional en español del YSR/11-18 (Achenbach \& Rescorla, 2001) al español de México. La sección de 112 ítems Likert del YSR/11-18 se piloteó en un grupo de 47 adolescentes escolares de 13 a 18 años. Se ajustó la redacción de cinco ítems por contener palabras desconocidas por los adolescentes (p. e. "tozudo" cambió a "necio"). Se entregó un cuadernillo con los instrumentos integrados y una hoja de respuesta para lectora óptica. Los adolescentes contestaron de manera anónima. La aplicación fue grupal con la colaboración de cuatro psicólogos y un supervisor. Con base en los lineamientos éticos del Acta de Helsinki (Comisión Nacional para la Protección de los Sujetos Humanos de Investigación Biomédica y del Comportamiento, 2010), se entregaron consentimientos y asentimientos informados a las autoridades escolares, a los padres y a los adolescentes.

\section{Análisis de datos}

Se analizaron las características psicométricas de los ítems como sesgo y curtosis, su capacidad de discriminación, y el grado de correlación, a fin de reducir elementos, previamente al análisis factorial exploratorio como lo sugieren otros estudios (García, Rivera, Reyes, \& Díaz-Loving, 2006; Roque, Acle, \& García, 2009). Se seleccionaron los ítems con curtosis y sesgo $\leq 1.5$; con valores $r$ entre .200 y $.800(p \leq 05)$ y valores $t$ de Student $(p \leq 05)$ (Lloret et al., 2014). Los coeficientes de Kaiser-Meyer-Olkin $(K M O)$, y el de prueba de esfericidad de Barlett $\left(X^{2}\right)$ se verificaron para valorar la pertinencia de un análisis factorial exploratorio (Ferrando \& Anguiano, 2010). Se realizó el análisis factorial exploratorio (AFE) por el método de componentes principales $(\mathrm{ACP}=$ Análisis por Componentes Principales) con rotación ortogonal (varimax) (Nunnally \& Bernstein, 1995) con la muestra 1 $(n=395)$, a fin de conocer la estructura del YSR/11-18 en este estudio. Con la muestra 2 $(n=395)$ se corrió un análisis factorial confirmatorio (AFC) para ratificar la estructura obtenida con el AFE. Se consideraron como indicadores de ajuste del modelo, los valores $X^{2}$ (CMNI) y la razón de $X^{2}$ /grados de libertad
(CMNI/DF) como medidas de la parsimonia. Se obtuvo el índice de bondad de ajuste (GFI) aceptando como buen ajuste un valor $>.90$; el error cuadrático medio de la aproximación (RMSEA), cuyos valores <.08 indican buen ajuste (óptimos <.05); los índices incrementales como AGFI o índice de bondad de ajuste corregido, el TLI (índice de Tucker-Lewis), y el CFI o índice de bondad de ajuste comparativo, cuyos valores cercanos a 1, indican un ajuste adecuado, considerado como un mejor indicador de ajuste que $X^{2}$ en muestras con $\mathrm{N}>200$ (Byrne, 2010; Ullman, 2006). La confiabilidad se evaluó a través del índice de consistencia interna alfa de Cronbach, tanto para el análisis de ítems (elemento-escala) como para cada uno de los factores resultantes. Se corrieron análisis de correlación entre factores. El análisis de datos se realizó con las versiones 21 de SPSS y del AMOS (Arbuckle, 2012).

\section{Resultados}

\section{Análisis factorial exploratorio}

Con base en el análisis de ítems, se seleccionaron 64 ítems para el AFE, debido a que 48 ítems presentaron las siguientes características: a. asimetría y curtosis $>1.500$; b. coeficientes $r<.200$ o $r>.800$; c. valores $t$ de Student con $p>.05$. Para el AFE, se siguieron cuatro criterios para la elección de ítems: 1.cargas factoriales por ítem $\geq .400 ; 2$. coeficientes alfas de Cronbach $>.60 ; 3$. ítems con cargas en un solo factor; y 4. mínimo tres ítems por factor (Nunnally \& Bernstein, 1995). Los datos de adecuación muestral de Kaiser-Mayer-Oklin $(K M O=.857)$ y el indicador de esfericidad de Bartlett $\left(X^{2}=10558.338 ; p=.000\right.$; $g l=1275)$ fueron aceptables indicando la pertinencia de un análisis factorial.

El análisis por $\mathrm{CP}$ con dichos criterios agrupó 50 ítems en 12 factores que en conjunto explicaron el 55.54\% de la varianza. La estructura factorial, cargas factoriales y el porcentaje de varianza se presentan en la Tabla 2 conforme al porcentaje decreciente de varianza explicada por factor: 1. Depresión, que refiere sentimientos de tristeza y que afectan al propio individuo; 2. Ruptura de Reglas, conductas que implican conducta disruptiva que genera malestar en otros; 
Tabla 2. Estructura factorial del YSR/11-18

\begin{tabular}{|c|c|c|c|}
\hline Factor & Ítems & $\begin{array}{c}\text { Carga } \\
\text { factorial }\end{array}$ & $\begin{array}{l}\text { Varianza } \\
\text { explicada }\end{array}$ \\
\hline \multirow[t]{6}{*}{ 1. Depresión (D) } & 9. No puedo quitarme de la mente ciertos pensamientos & .639 & $16.75 \%$ \\
\hline & 12. Me siento muy solo(a) & .662 & \\
\hline & 13. Me siento confundido(a) o como si estuviera en las nubes & .620 & \\
\hline & 14. Lloro mucho & .657 & \\
\hline & 27. Tengo celos de otras personas & .564 & \\
\hline & 103. Me siento infeliz, triste o deprimido(a) & .635 & \\
\hline \multirow{9}{*}{$\begin{array}{l}\text { 2. Ruptura } \\
\text { de Reglas (RR) }\end{array}$} & 2. Bebo alcohol sin permiso de mis padres & 639 & $7.84 \%$ \\
\hline & 22. Desobedezco a mis padres & .662 & \\
\hline & 23. Desobedezco en la escuela & .620 & \\
\hline & 28. Rompo las reglas en casa, en la escuela, o en otro lugar & 657 & \\
\hline & 37. Peleo mucho & .564 & \\
\hline & 39. Me junto con los jóvenes que se meten en problemas & .635 & \\
\hline & 41. Actúo sin pensar & 639 & \\
\hline & 43. Digo mentiras o engaño a los demás & .662 & \\
\hline & 92. Me gusta hacer reír a los demás & .708 & $6.39 \%$ \\
\hline \multirow{4}{*}{$\begin{array}{l}\text { 3. Conducta } \\
\text { Prosocial/Sentido del } \\
\text { Humor }(\mathrm{CP} / \mathrm{SH})\end{array}$} & 98. Me gusta ayudar a otras personas & .708 & \\
\hline & 107. Me gusta un buen chiste & .660 & \\
\hline & 108. Me gusta llevar las cosas con calma & .640 & \\
\hline & 109. Trato de ayudar a los demás cuando puedo & .644 & \\
\hline \multirow{5}{*}{ 4. Retraimiento (R) } & 65. Me rehúso a hablar & .636 & $3.73 \%$ \\
\hline & 69. Soy muy reservado(a); me callo todo & .571 & \\
\hline & 71. Me cohíbo, me avergüenzo con facilidad & .600 & \\
\hline & 75. Soy demasiado tímido(a) & .761 & \\
\hline & 111. Evito relacionarme con los demás & .426 & \\
\hline 5. Autoconcepto & 59. Puedo ser bastante amigable & .654 & $3.39 \%$ \\
\hline \multirow[t]{3}{*}{ Positivo (AP) } & 60. Me gusta tratar cosas nuevas & .715 & \\
\hline & 73. Puedo trabajar bien con mis manos & .464 & \\
\hline & 88. Me gusta estar con otras personas & .608 & \\
\hline \multirow{4}{*}{$\begin{array}{l}\text { 6. Quejas Somáticas } \\
\text { (QS) }\end{array}$} & 51. Me siento mareado(a) & .627 & \\
\hline & 54. Me siento demasiado cansado(a) sin ninguna buena razón & .409 & $3.20 \%$ \\
\hline & 56b. Dolores de cabeza & .796 & \\
\hline & 56f. Dolores de estómago & .578 & \\
\hline \multirow{3}{*}{$\begin{array}{l}\text { 7. Problemas de } \\
\text { Pensamiento (PP) }\end{array}$} & 17. Sueño despierto(a) a menudo & .431 & $2.78 \%$ \\
\hline & 84. Hago cosas que otras personas piensan que son extrañas & .822 & \\
\hline & 85. Tengo ideas que otras personas pensarían que son extrañas & .832 & \\
\hline \multirow{3}{*}{$\begin{array}{l}\text { 8. Problemas } \\
\text { de Sueño (PS) }\end{array}$} & 76. Duermo menos que la mayoría de los jóvenes & .668 & $2.55 \%$ \\
\hline & 100. No duermo bien & .680 & \\
\hline & 102. Tengo poca energía & .475 & \\
\hline \multirow{3}{*}{$\begin{array}{l}\text { 9. Problemas } \\
\text { de Atención (PA) }\end{array}$} & 8. No puedo concentrarme o prestar atención por mucho tiempo & .685 & $2.47 \%$ \\
\hline & 10. No puedo estarme quieto(a) & .525 & \\
\hline & 78. No presto atención o me distraigo fácilmente & 689 & \\
\hline \multirow[t]{3}{*}{ 10. Ansiedad (A) } & 44. Me muerdo las uñas & .773 & $2.28 \%$ \\
\hline & 45. Soy nervioso (a), tenso (a) & .671 & \\
\hline & 50. Soy demasiado ansioso(a) o miedoso(a) & .566 & \\
\hline \multirow{3}{*}{$\begin{array}{l}\text { 11. Búsqueda } \\
\text { de Atención (BA) }\end{array}$} & 68. Grito mucho & .637 & $2.14 \%$ \\
\hline & 93. Hablo demasiado & .534 & \\
\hline & 104. Soy más ruidoso(a) que otros(as) personas & .539 & \\
\hline \multirow{3}{*}{$\begin{array}{l}\text { 12.Conducta Agresiva } \\
\text { (AG) }\end{array}$} & 3. Discuto mucho & .448 & $2.02 \%$ \\
\hline & 16. Soy malo(a) con los demás & .635 & \\
\hline & 95. Me enfado con facilidad & .556 & \\
\hline Total & 50 ítems & & $55.54 \%$ \\
\hline
\end{tabular}




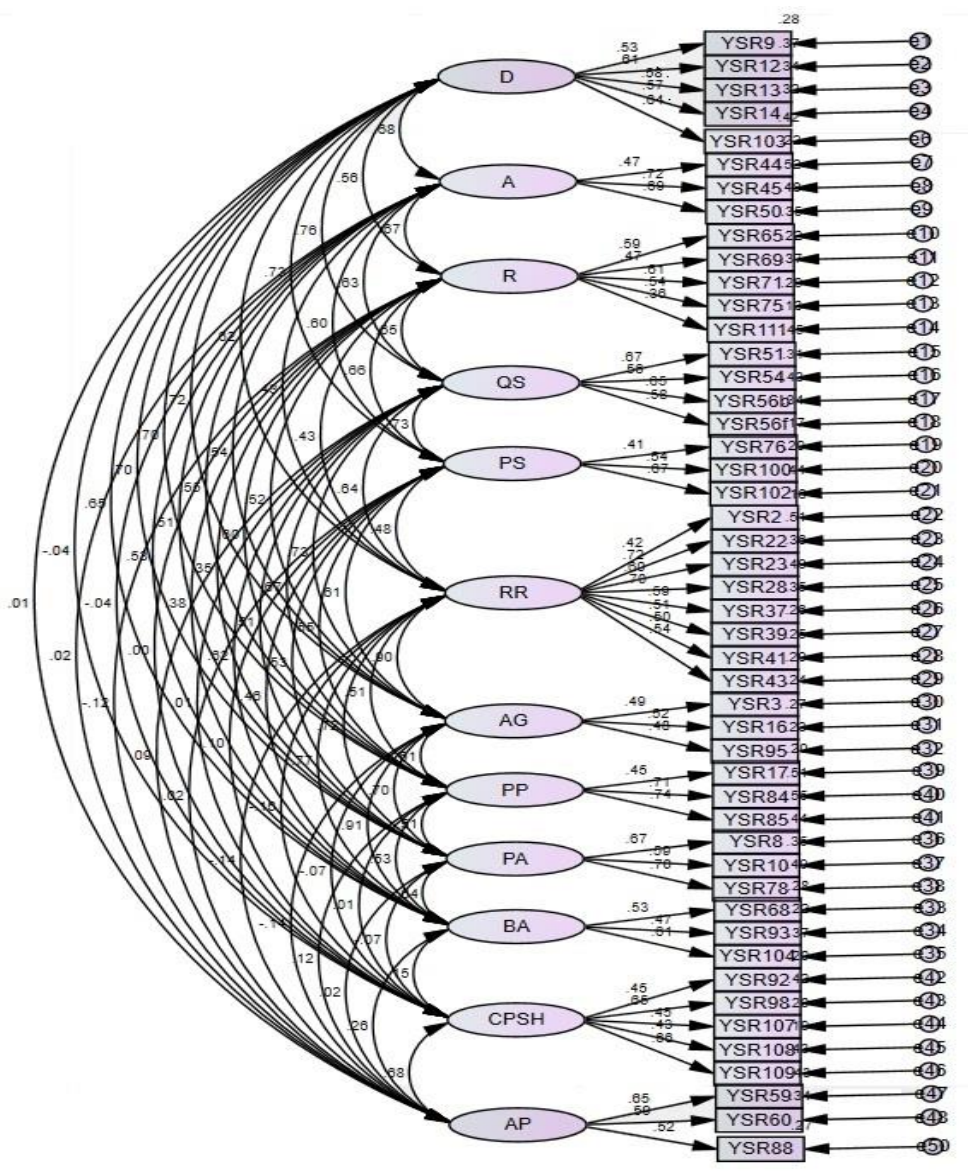

Figura 1. Modelo del AFC de primer orden con doce factores de Banda Estrecha del YSR/11-18

3. Conducta Prosocial/Sentido del Humor, que identifica comportamientos asociados con buen humor y acciones orientadas a apoyar a otros; 4. Retraimiento, que hace referencia a conductas de evitación de interacción social; 5. Autoconcepto Positivo, refiere a la percepción positiva de sí mismo; 6. Quejas Somáticas, identifica la presencia de síntomas somáticos como dolores de cabeza, de estómago, entre otros; 7. Problemas de Pensamiento, que identifica presencia de ideas bizarras; 8. Problemas de Sueño, que valora la presencia de trastornos del sueño; 9. Problemas de Atención, que evalúa dificultad de concentración; 10. Ansiedad, que implica preocupaciones; 11. Búsqueda de Atención, que refiere la necesidad de llamar la atención; 12. Conducta Agresiva, que implica comportamiento disruptivo o antisocial que afecta a terceros.

\section{Análisis factorial confirmatorio}

El ajuste del modelo de doce factores del YSR/11-18 se evaluó a través de un AFC, utilizando el método de Máxima Verosimilitud (Arbuckle, 2012), utilizando el AMOS del SPSS
21. Se asumió un modelo multifactorial y la existencia de doce variables latentes con covarianzas entre los errores. Todos los índices de bondad del ajuste del modelo fueron adecuados y se encuentran dentro de los márgenes aceptados: RMSEA=.038 (.02-.08); CMIN/DF=1.58; GFI=.851; AGFI $=.829 ; \mathrm{TLI}=.853 ; \mathrm{CFI}=.867 ; X^{2}=1750.700[(90$, $\mathrm{n}=395), p<.01]$. Todas las saturaciones factoriales estimadas para el modelo resultaron estadísticamente significativas $(p \leq .01)$, apoyando la validez factorial (Kline, 2005). El RMSEA fue <.05, confirmando un ajuste adecuado e incluso óptimo del modelo (Lloret et al., 2014). El CMIN/DF fue <3 lo que indica un adecuado ajuste (Ullman, 2006). El valor de GFI también refleja un ajuste aceptable del modelo (Ferrando \& Anguiano, 2010). Asimismo los índices incrementales (AGFI, TLI y CFI) son próximos a 1 , aunque marginalmente por debajo de lo óptimo recomendado (>.90), revelan un ajuste aceptable (Byrne, 2010). Los valores de $X^{2}$ alrededor de 2 son indicadores de ajuste, aunque el valor de $p$ fue significativo. El modelo final de 48 ítems (eliminando cuatro con pesos factoriales bajos en el AFE), mostró un ajuste aceptable (Figura 1). 


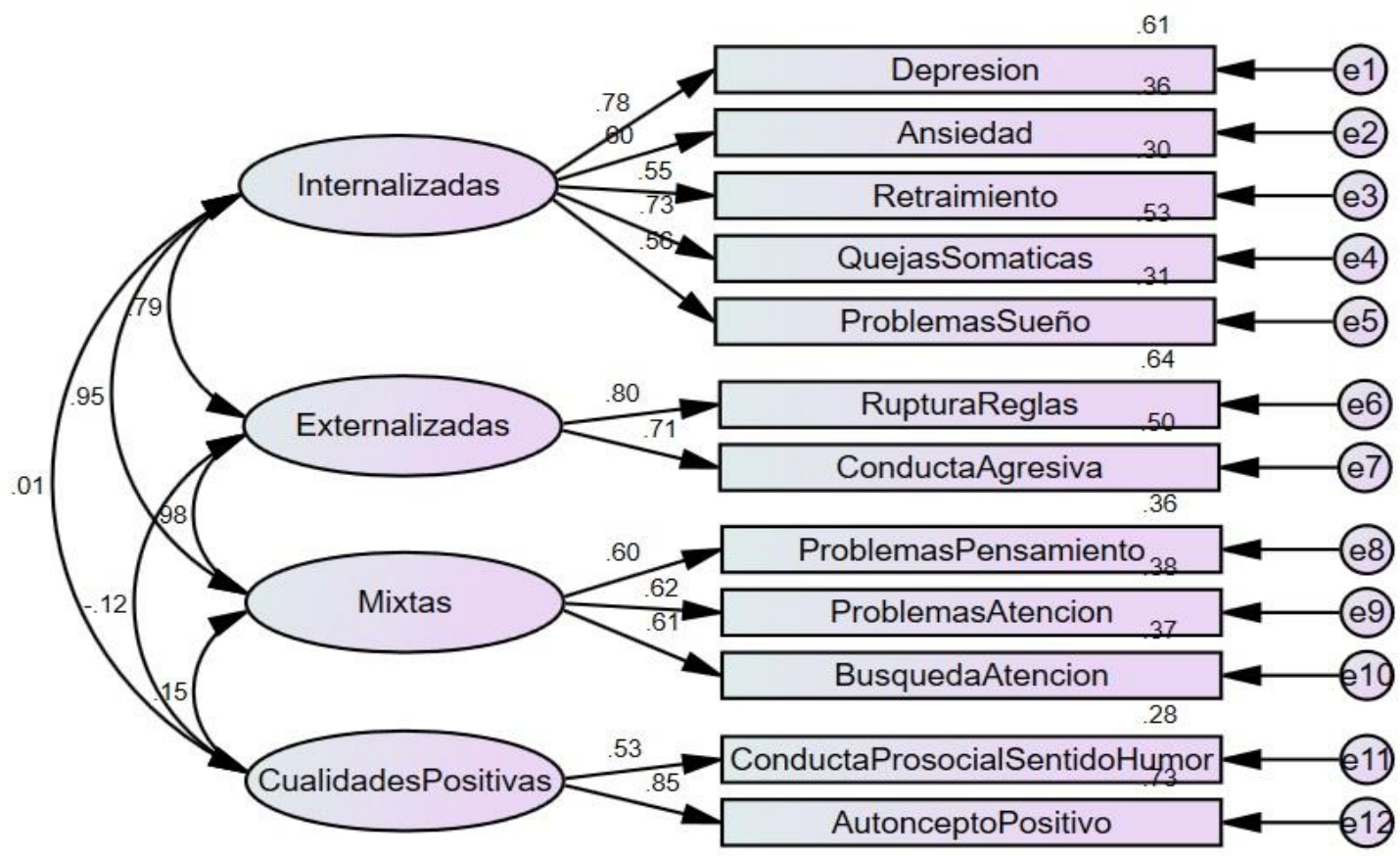

Figura 2. Modelo del AFC de segundo orden de cuatro factores de Banda Ancha y doce de Banda Estrecha del YSR/11-18

El ajuste del modelo de cuatro factores de segundo orden o de Banda Ancha (Achenbach \& Rescorla, 2001; Rescorla et al., 2007), se evaluó a través de un AFC, utilizando el método de Máxima Verosimilitud (Arbuckle, 2012). Los estimadores indican un buen ajuste en general: RMSEA=.064 (.050-.078); AGFI=.923; GFI=.953; $\mathrm{CMIN} / \mathrm{DF}=2.462 ; \mathrm{TLI}=.934 ; \mathrm{CFI}=.948 ; X^{2}=118.200$ [(90, $\mathrm{n}=395), p<.01]$, en especial uno de los más robustos como el RMSEA, y con valores mayores de .90 en los índices incrementales como se recomienda (Byrne, 2010; Ullman, 2006).

El análisis factorial de segundo orden reagrupó los doce factores de $\mathrm{BE}$ en cuatro factores de BA: 1. Problemas Internalizados: Depresión, Ansiedad, Quejas Somáticas, Problemas de Sueño y Retraimiento (factor compuesto por ítems de Depresión y Retraimiento); 2. Problemas Externalizados: Ruptura de Reglas y Conducta Agresiva; 3. Problemas Mixtos: conformado por Problemas de Pensamiento y Problemas de Atención, además de Búsqueda de Atención; y 4. Cualidades Positivas: surgió como un nuevo factor, producto de la agrupación de dos nuevos factores de primer orden relacionados con aspectos positivos: Conducta Prosocial/Sentido del Humor y Autoconcepto Positivo, confirmándose la estructura inicial de BE arrojada por el ACP (Figura 2).

La Tabla 3 muestra la estructura final de los factores de primer orden (Banda Estrecha), de segundo orden (Banda Ancha), el número de ítems que los componen, su definición conceptual, y los coeficientes alfa de Cronbach (de $\alpha=.608$ a $\alpha=.942$ ), que indican una consistencia interna total y por factores, adecuada.

\section{Análisis correlacional entre factores}

La Tabla 4 muestra correlaciones positivas significativas $(p \leq .01)$ entre la mayoría de los factores, que van de $r=.119$ a $r=.525$, que reflejan independencia de los factores. Se observaron pocas correlaciones negativas significativas $(p<.01)$ cuyos valores oscilaron entre $r=-.111 \mathrm{y}$ $r=-.159$, por ejemplo, entre Conducta Prosocial/Sentido del Humor y Problemas de Pensamiento, así como Autoconcepto Positivo y Búsqueda de Atención, aunque con el resto de factores no sé encontró relación. Por otra parte, Conducta Prosocial/Sentido del Humor presentó correlaciones en general bajas, aunque significativas con la mayoría de los otros factores, excepto con Autoconcepto Positivo con el que se observa un valor $r$ positivo moderadamente alto. No se encontraron correlaciones significativas con Retraimiento, Quejas Somáticas, Problemas de 
Tabla 3. Factores de Banda Estrecha y Banda Ancha del YSR/11-18: número de ítems, definiciones, y alfas de Cronbach $(\alpha)$

\begin{tabular}{|c|c|c|c|}
\hline Factores & $\begin{array}{l}\text { No. de } \\
\text { ítems }\end{array}$ & Definición & $\alpha$ \\
\hline $\begin{array}{l}\text { Problemas } \\
\text { Internalizados }\end{array}$ & 21 & $\begin{array}{c}\text { Elevado control de la emoción y estado de ánimo alterado que } \\
\text { implica malestar a sí mismo. }\end{array}$ & .779 \\
\hline Depresión & 6 & Sentimientos de inferioridad, tristeza y preocupación. & .782 \\
\hline Ansiedad & 3 & Conductas que se asocian con miedo y nerviosismo. & .654 \\
\hline Retraimiento & 5 & $\begin{array}{c}\text { Conductas de aislamiento, tendencia a evitar convivir con las } \\
\text { personas. }\end{array}$ & 690 \\
\hline Quejas Somáticas & 4 & Problemas físicos y agotamiento sin causa medica aparente. & 693 \\
\hline $\begin{array}{l}\text { Problemas de } \\
\text { Sueño }\end{array}$ & 3 & Alteraciones relacionadas con procesos del sueño. & .608 \\
\hline $\begin{array}{l}\text { Problemas } \\
\text { Externalizados }\end{array}$ & 11 & $\begin{array}{l}\text { Bajo control de comportamientos que implican conflictos y riesgo } \\
\text { para otras personas. }\end{array}$ & .762 \\
\hline Ruptura de Reglas & 8 & $\begin{array}{c}\text { Conductas asociadas al consumo de sustancias, engaño y } \\
\text { problemas para apegarse a las normas. }\end{array}$ & .776 \\
\hline Conducta Agresiva & 3 & $\begin{array}{l}\text { Conductas que implican agresión física como discutir y pelear con } \\
\text { otras personas. }\end{array}$ & .654 \\
\hline Problemas Mixtos & 9 & $\begin{array}{c}\text { Problemas que comparten características de los problemas } \\
\text { internalizados y externalizados. }\end{array}$ & 699 \\
\hline $\begin{array}{l}\text { Problemas de } \\
\text { Pensamiento }\end{array}$ & 3 & Presencia de ideas extrañas o bizarras, tendencia a la fantasía & .664 \\
\hline $\begin{array}{l}\text { Problemas de } \\
\text { Atención }\end{array}$ & 3 & $\begin{array}{c}\text { Dificultad para terminar actividades, prestar atención y/o } \\
\text { mantenerse quieto. }\end{array}$ & 642 \\
\hline $\begin{array}{l}\text { Búsqueda de } \\
\text { atención }\end{array}$ & 3 & $\begin{array}{c}\text { Comportamiento encaminado a llamar la atención de otras } \\
\text { personas. }\end{array}$ & .661 \\
\hline Cualidades Positivas & 9 & $\begin{array}{c}\text { Características personales que se asocian con la adaptación } \\
\text { positiva. }\end{array}$ & .745 \\
\hline $\begin{array}{l}\text { Conducta Prosocial } \\
\text { / Sentido del Humor }\end{array}$ & 5 & $\begin{array}{l}\text { Comportamiento orientado a apoyar a otros con consecuencias } \\
\text { socialmente positivas. }\end{array}$ & .775 \\
\hline $\begin{array}{l}\text { Autoconcepto } \\
\text { Positivo }\end{array}$ & 4 & Imagen positiva que tiene el adolescente de sí mismo. & .661 \\
\hline Total ítems & 50 & & .949 \\
\hline
\end{tabular}

Tabla 4. Correlaciones entre los factores de primer orden o Banda Estrecha del YSR/11-18

\begin{tabular}{|c|c|c|c|c|c|c|c|c|c|c|c|}
\hline Factores & 2 & 3 & 4 & 5 & 6 & 7 & 8 & 9 & 10 & 11 & 12 \\
\hline 1. Depresión & $.370 * *$ & .045 & $.445 * *$ & -.224 & $.487 * *$ & $.388 * *$ & $.337 * *$ & $.369 * *$ & $.418 * *$ & $.222 * *$ & $.348 * *$ \\
\hline $\begin{array}{l}\text { 2. Ruptura de } \\
\text { Reglas }\end{array}$ & - & -.036 & $.119 * *$ & .061 & $.318 * *$ & $.159 * *$ & $.189 * *$ & $.423 * *$ & $.220 * *$ & $.317 * *$ & $.520 * *$ \\
\hline $\begin{array}{l}\text { 3.Conducta } \\
\text { Prosocial/Sentido } \\
\text { del Humor }\end{array}$ & & - & .048 & $.525 * *$ & .085 & $-127 * *$ & $-111 * *$ & .042 & $.131^{* *}$ & $.239 * *$ & .025 \\
\hline 4. Retraimiento & & & - & -.064 & $.309 * *$ & $.278 * *$ & $.326 * *$ & $.234 * *$ & $.377 * *$ & .045 & $.185^{* *}$ \\
\hline $\begin{array}{l}\text { 5. Autoconcepto } \\
\text { Positivo }\end{array}$ & & & & - & .061 & .002 & .039 & .026 & .045 & $-159 * *$ & .037 \\
\hline $\begin{array}{l}\text { 6. Quejas } \\
\text { Somáticas }\end{array}$ & & & & & - & $.319 * *$ & $.436^{* *}$ & $.287 * *$ & $.410 * *$ & $.196 * *$ & $.277 * *$ \\
\hline $\begin{array}{l}\text { 7. Problemas de } \\
\text { Pensamiento }\end{array}$ & & & & & & - & $.325 * *$ & $.305 * *$ & $.242 * *$ & $.228 * *$ & $.229 * *$ \\
\hline $\begin{array}{l}\text { 8. Problema de } \\
\text { Sueño }\end{array}$ & & & & & & & - & $.241 * *$ & $.302 * *$ & $.213 * *$ & $.179 * *$ \\
\hline $\begin{array}{l}\text { 9. Problemas de } \\
\text { Atención }\end{array}$ & & & & & & & & - & $.268 * *$ & $.393 * *$ & $.364 * *$ \\
\hline 10. Ansiedad & & & & & & & & & - & $.238 * *$ & $.227 * *$ \\
\hline $\begin{array}{l}\text { 11. Búsqueda de } \\
\text { Atención } \\
\text { 12. Conducta } \\
\text { Agresiva }\end{array}$ & & & & & & & & & & - & $.397 * *$ \\
\hline
\end{tabular}


Tabla 5. Descriptivos muestra total y diferencias de medias por sexo en los factores de Banda Estrecha y Banda Ancha del YSR/11-18

\begin{tabular}{|c|c|c|c|c|c|c|c|c|}
\hline & \multicolumn{2}{|c|}{$\begin{array}{c}\mathrm{MT} \\
\mathrm{N}=790\end{array}$} & \multicolumn{2}{|c|}{$\begin{array}{l}\text { Chicos } \\
n=387\end{array}$} & \multicolumn{2}{|c|}{$\begin{array}{l}\text { Chicas } \\
n=403\end{array}$} & \multirow[b]{2}{*}{$t$} & \multirow[b]{2}{*}{$p$} \\
\hline & $M$ & $D E$ & $M$ & $D E$ & $M$ & $D E$ & & \\
\hline Problemas Internalizados & .55 & .33 & .44 & .28 & .63 & .34 & -7.25 & $<.001$ \\
\hline Depresión & .53 & .46 & .38 & .37 & .64 & .49 & -6.93 & $<.001$ \\
\hline Ansiedad & .77 & .57 & .60 & .50 & .90 & .58 & -.635 & $<.001$ \\
\hline Retraimiento & .52 & .43 & .47 & .40 & .54 & .45 & -2.46 & .054 \\
\hline Quejas Somáticas & .50 & .45 & .37 & .39 & .60 & .47 & -6.13 & $<.001$ \\
\hline Problemas de Sueño & .51 & .48 & .41 & .44 & .59 & .50 & -4.30 & $<.001$ \\
\hline Problemas Externalizados & .55 & .31 & .58 & .32 & .53 & .32 & -2.71 & .050 \\
\hline Ruptura de Reglas & .46 & .36 & .46 & .36 & .46 & .37 & -.188 & .906 \\
\hline Conducta Agresiva & .68 & .48 & .72 & .47 & .64 & .48 & -2.45 & .064 \\
\hline Problemas Mixtos & .59 & .46 & .51 & .40 & .65 & .45 & -219 & .013 \\
\hline $\begin{array}{l}\text { Problemas de } \\
\text { Pensamiento }\end{array}$ & .55 & .54 & .49 & .52 & .61 & .55 & -2.58 & .010 \\
\hline $\begin{array}{l}\text { Problemas de } \\
\text { Atención }\end{array}$ & .65 & .51 & .60 & .49 & .69 & .53 & -2.13 & .033 \\
\hline $\begin{array}{l}\text { Búsqueda de } \\
\text { Atención }\end{array}$ & .57 & .48 & .44 & .43 & .66 & .49 & -5.44 & $<.001$ \\
\hline Cualidades Positivas & 1.28 & .45 & 1.25 & .46 & 1.31 & .43 & -1.44 & .151 \\
\hline $\begin{array}{l}\text { Conducta Prosocial } \\
\text { /Sentido del Humor }\end{array}$ & 1.24 & .51 & 1.20 & .537 & 1.27 & .50 & -1.56 & .118 \\
\hline $\begin{array}{l}\text { Autoconcepto } \\
\text { Positivo }\end{array}$ & 1.34 & .61 & 1.31 & .53 & 1.35 & .50 & -.877 & .381 \\
\hline
\end{tabular}

Atención y Conducta Agresiva. Por último se observa que Depresión, aunque presentó las correlaciones más altas con casi todos los factores, particularmente con Quejas Somáticas y Retraimiento; no presentó correlación con Conducta Prosocial/Sentido del Humor y Autoconcepto Positivo.

\section{Análisis comparativos por sexo}

En la Tabla 5 se observa que las chicas presentan valores más altos en Problemas Internalizados con diferencias estadísticamente significativas $(p<.001)$ con los hombres, excepto en Retraimiento. En Problemas Mixtos, también se observan diferencias por sexo, con significancia estadísticas $(p \leq .05$ y $p<.001)$, con mayores puntuaciones en las mujeres. Aunque los hombres presentan más Problemas Externalizados que las mujeres las diferencias no son estadísticamente significativas. En contraste, las chicas presentaron puntuaciones discretamente superiores a los chicos en Cualidades Positivas, aunque tampoco son significativas.

\section{Discusión}

El objetivo del estudio fue analizar las propiedades psicométricas de la versión oficial internacional en español del YSR/11-18 (Achenbach \& Recorla, 2001), dada la evidencia a nivel mundial de su utilidad en la identificacion de Problemas Internalizados, Externalizados, Mixtos y Cualidades Positivas. Se optó por correr nuevamente un ACP, dadas las variaciones de la estructura previamente reportadas, principalmente con adolescentes de habla hipana y en México (Betancourt, 2007; Betancourt \& Andrade, 2010; Bordin et al., 2013; Valencia \& Andrade, 2005). La solución factorial encontrada en este estudio presenta similitudes con el modelo original y con algunas estructuras reportadas en otros países (Ivanova et al., 2007; Lemos et al., 2002; Rescorla et al., 2007; 2012), en contraste a lo reportado con adolescentes mexicanos con la versión modificada (Betancourt, 2007; Valencia \& Andrade, 2005). En la presente investigación, Problemas Internalizados mantuvo los mismos ítems aunque Depresión/Ansiedad se factorizó en Ansiedad y Depresión, de manera similar a los hallazgos de 
Lemos y colaboradores (2002), lo que podría resultar de mayor utilidad para el diseño de intervenciones indicadas. Problemas de Sueño emergió como un nuevo factor de Problemas Internalizados lo que permite diferenciar trastornos fisiológicos, ansiedad y depresión. Asimismo puede tipificarse como un problema específico independientemente de la ansiedad y depresión, lo cual sirve de base para la elección de un tratamiento más preciso. Los factores de Banda Estrecha correspondiente a los Problemas Externalizados no sufrieron modificaciones, lo cual confirma la mayor estabilidad de este grupo de conductas como se reporta (Bordin et al., 2013; Ivanova et al., 2007; Rescorla et al., 2007; 2012).

Problemas de Atención y Problemas de Pensamiento se integraron en Problemas Mixtos como se reporta en la investigación previa (Abad et al., 2000; Ivanova et al., 2007; Lemos et al., 1992; 2002; Rescorla et al., 2007; 2012). Los ítems de Problemas Sociales (p. e. "me siento solo") se agruparon en Retraimiento, lo que resultó conceptualmente más claro lo cual coincide en parte con los datos de Daset y colaboradores (2009). Búsqueda de Atención emergió a partir de ítems que implican conductas de demanda social ( $p$.e. "hablo demasiado") de forma similar a lo reportado con diversos grupos de adolescentes españoles (Lemos et al., 1992a; 1992b; 2002).

La factorización de Autoconcepto Positivo y de Conducta Prosocial/Sentido del Humor en una dimensión que se denominó Cualidades Positivas, fue resultado de la agrupación de ítems que conllevan características positivas y conducta prosocial, previamente explorados en adolescentes españoles (Lemos et al., 1992a; 1992b). Estos datos también se ajustan a los de Daset y colaboradores (2009) quienes informan sobre un Síndrome Prosocial, así como de otras investigaciones que muestran características positivas (Abad et al., 2000; Rescorla et al., 2007; Zubeidat et al., 2009). Llama la atención que escasamente se mencione este tipo de características a pesar de que representan recursos que pueden constituir la base para la intervención.

La obtención de factores adicionales a la estructura presentada por Achenbach (Achenbach, 1991; Achenbach \& Recorla, 2001), en el presente estudio, reproduce en general los resultados en
España y Latinoamérica (Abad et al., 2000; Betancourt \& Andrade, 2010; Daset et al., 2009; Lemos et al., 1992a; Sandoval et al., 2006; Zubeidat et al., 2009), quizá debido, por un lado, a las similitudes entre población adolescente de habla hispana, y una mayor diferencia cultural con adolescentes anglosajones y de otros países. No obstante, contar con factores nuevos de Banda Estrecha como Problemas de Sueño y Búsqueda de Atención, y de Banda Ancha como el de Cualidades Positivas representa una posible ventaja para el diagnóstico diferencial, la valoración de fortalezas y el tratamiento integral. Los índices de consistencia interna más elevada para los factores de Problemas Internalizados y Externalizados confirma su solidez en varios contextos (Ivanova et al., 2007; Rescorla et al., 2007; 2012). Sin embargo, los factores de Cualidades Positivas y Problemas Mixtos, presentaron los índices más bajos como se advierte previamente (Rescorla et al., 2007). Aunque satisfactorios (Nunnally \& Bernstein, 1995), se recomienda explorar su consistencia interna en otras muestras y valorar su pertinencia.

Los resultados del AFC (Ullman, 2006), confirmaron una estructura de doce factores con 48 ítems, dos menos que en la solución del AFE, debido a los estimadores que indicaban la conveniencia de su eliminación. El modelo resultante reveló que todos los índices de ajuste son adecuados, a excepción del valor de $X^{2}$ que aunque dentro del rango aceptable resultó significativo, lo cual podría representar una limitación, debido al tamaño de la muestra. Como advierten algunos autores (Byrne, 2010; Ullman, 2006) los valores de $X^{2}$ suelen ser significativos en las muestras grandes, por lo que se utilizan varios indicadores de ajuste. El CFI y el RMSEA, son de los indicadores más fuertes, siendo el primero el que se recomienda como otra opción a la $X^{2}$ para probar el ajuste de un modelo (Ullman, 2006). De esta forma, los indicaron en general indicaron buen ajuste del YSR/11-18, para esta muestra, presentando una estructura parsimoniosa y conceptualmente razonable. No obstante, un próximo estudio podría utilizar muestras obtenida con métodos más rigurosos de selección.

Asimismo, la agrupación de los factores de primer orden (Banda Estrecha) y segundo orden (Banda Ancha), confirma por un lado la estructura 
de Achenbach y Rescorla (2001), y por otro, presenta congruencia teórica al mostrar más factores de BE cuya agrupación corresponde a la separación de comportamientos que nosológicamente pueden ser diferentes como ansiedad y depresión (Achenbach, 2015). Aunque el surgimiento de más factores, podría contravenir la ley de la parsimonia (Lloret et al., 2014), la disminución de la mitad de ítems, apoya la idea de que podría resultar útil para la práctica clínica con fines diagnósticos y de intervención indicada, así como una medida de tamizaje para identificar tempranamente problemas desde el ámbito escolar. La evidencia muestra que los Problemas Internalizados como la Depresión y Ansiedad comparten sintomatología, aunque también pueden presentarse como cuadros diferenciados (como en esta muestra) que podrían relacionarse independientemente con retraimiento $o$ con alteraciones psicofisiológicas. Por otro lado, el grupo de Problemas Externalizados, compuesto por Ruptura de Reglas y Conducta Agresiva, se mantuvo de manera consistente en el AFE y en el AFC, como en otros estudios (Ivanova et al., 2007; Rescorla et al., 2004). En esta muestra, Problemas de Pensamiento, Problemas de Atención y Búsqueda de Atención, se agruparon en lo que Achenbach y Rescorla (2001) denominan Problemas Mixtos por no quedar agrupados en ningún otro factor. El surgimiento y agrupación de Conductas Prosociales y Autoconcepto Positivo en el factor de Banda Ancha de Cualidades Positivas resulta similar a lo reportado en estudios transculturales con el YSR/11-18 más recientes (Rescorla et al., 2007), así como en Latinoamérica (Bordin et al., 2013; Daset et al., 2009) lo que tiene implicaciones para las acciones preventivas y comparación entre diferentes países. Las correlaciones de moderadamente bajas a altas moderadas entre los factores, indican que las variables que explora el modelo resultante, son suficientemente independientes (Lloret et al., 2014), apoyando la pertinencia de las diversas dimensiones y factores que exploran diferentes constructos relacionados con la adaptación que en conjunto permitirían evaluar de manera integral a población adolescente.

Las diferencias por sexo refieren una tendencia mayor de las chicas a presentar
Problemas Internalizados que los chicos, quienes a su vez presentan ligeramente más Problemas Externalizados, lo que resulta consistente parcialmente con otros estudios (Abad et al., 2000; Ivanova et al., 2007; Rescorla et al., 2007; 2012; Sandoval et al., 2006), ya que en esta muestra no hubo diferencias estadísticamente significativas. Asimismo indican que las chicas están presentando mayores niveles de conducta antisocial lo que coincide con informes previos (Alarcón \& Bárrig, 2015; Daset et al., 2009). A pesar de que las diferencias por sexo se centraron en los Problemas Internalizados, sería recomendable que la investigación futura realizara análisis factoriales por sexo e incluso por rangos de edad.

En síntesis, los datos indican que el YSR/1118 presenta una estructura factorial con indicadores de validez de constructo adecuados que lo convierten en un instrumento potencialmente útil para la valoración integral de la salud mental y el funcionamiento adolescente en población mexicana. El hecho de que se haya utilizado la versión oficial internacional en español plantea la posibilidad de contrastar los datos de población de habla hispana así como de otras culturas (Verhulst \& Achenbach, 1995). No obstante, dadas las limitaciones de este estudio, por ejemplo, la representatividad de la muestra al ser por conveniencia, estos hallazgos podrían considerarse como evidencia preliminar de la validez del instrumento, por lo que considera conveniente realizar otros análisis de validez (p. e. validez concurrente) y valorar su funcionamiento en muestras de diferentes contextos. De forma adicional podrían aplicarse otros métodos analíticos (p. e. análisis discriminante) para evaluar la capacidad de discriminación óptima de algunos factores para identificar la presencia de problemas y magnitud de los mismos, así como utilizar otros programas (p. e. $M$ plus, Factor) para el análisis de la validez de constructo, a fin de subsanar algunas restricciones de este estudio. Finalmente, cabe resaltar que la evidencia empírica de la existencia de un factor de aspectos positivos en adolescentes mexicanos representa la posibilidad de identificar, no solo problemas, sino también recursos, lo cual tiene implicaciones para una intervención basada en un enfoque preventivo. 


\section{Agradecimientos}

Este estudio fue realizado gracias al financiamiento del PAPIIT IN305917 de la Dirección General de Asuntos del Personal Académico de la Universidad Nacional Autónoma de México. Se agradece a Raquel Rodríguez Alcántara su apoyo en el procesamiento de datos.

\section{Conflicto de intereses}

Las autoras declaran no tener ningún conflicto de intereses.

\section{Referencias}

Abad, J., Forns, M., Amador, J., \& Martorell, B. (2000). Fiabilidad y validez del Youth Self Report en una muestra de adolescentes. Psicothema, 12(1), 49-54.

Achenbach, T. M. (2015). Transdiagnostic heterogeneity, hierarchical dimensional models, and societal, cultural, and individual differences in the developmental understanding of psychopathology. European Child \& Adolescent Psychiatry, 24(12), 14191422. doi:10.1007/s00787-015-0795-0

Achenbach, T. M., Rescorla, L. A., \& Ivanova, M. Y. (2012). International epidemiology of child and adolescent psychopathology I: Diagnoses, dimensions, and conceptual issues. Journal of the American Academic Child \& Adolescent Psychiatry, 51(12), 1261-1272. doi.org/10.1016/j.jaac.2012.09.010

Achenbach, T. M., \& Rescorla, L. A. (2001). Manual for the ASEBA school-age forms \& profiles. Burlington, VT: ASEBA.

Alarcón, D., \& Bárrig, P. (2015). Conductas internalizantes y externalizantes en adolescentes. Liberabit, 21(2), 253-259.

Andrade, P., Betancourt, D., \& Vallejo, A. (2010). Escala para evaluar problemas emocionales y conductuales en adolescentes. Investigación Universitaria Multidisciplinaria, 9(9), 37-44.

Arbuckle, J. (2012). AMOS users guide version 21.0. Chicago, USA: Marketing Department, SPSS Incorporated.

Ato, M., López, J., \& Benavente, A. (2013). Un sistema de clasificación de los diseños de investigación en psicología. Anales de Psicología, 29(3),1038-1059.
Barcelata, B. (2016). Cédula sociodemográfica del adolescente y su familia. (PAPIIT IN303714). Ciudad de México: Facultad de Estudios Superiores Zaragoza, UNAM.

Betancourt, D. (2007). Control parental y problemas internalizados y externalizados en niños y adolescentes. (Tesis inédita de Doctorado). Universidad Nacional Autónoma de México. Facultad de Psicología, Ciudad de México.

Betancourt, D., \& Andrade, P. (2010). Evaluación de problemas internalizados y externalizados en adolescentes. En A. Rivera, R. DiazLoving, L. Reyes, A. Sánchez, \& M. Cruz (Eds.). La Psicología Social en México. XIII (pp. 1041-1048). Ciudad de México: AMEPSO.

Bordin, I. A., Rocha, M. M., Paula, C. S., Teixeira, M. C., Achenbach, T. M., Rescorla, L.,...Silvares, E. F. (2013). Child Behavior Checklist (CBCL), Youth Self-Report (YSR) and Teacher's Report Form (TRF): An overview of the development of the original and Brazilian versions. Cadernos de Saude Publica, 29(1), 13-28. doi.org/10.1590/S0102-311X2013000500004

Byrne, B. (2010). Structural equation modeling with AMOS: Basic concepts, applications, and programming. New York, USA: Routledge

Cicchetti, D., \& Rogosch, F. (2002). A developmental psychopathology perspective on adolescence. Journal Consulting Clinical Psychology, 70(1), 6-20. doi:10.1037//0022-006X.70.1.6

Comisión Nacional para la Protección de los Sujetos Humanos de Investigación Biomédica y del Comportamiento (2010). Principios y guías éticos para la protección de los sujetos humanos de investigación. Informe Belmont. Washington, D.C.: Departamento de Salud, Educación y Bienestar. Disponible en: http//: pcb.ub.edu/bioeticaidret/archivos/norm/Infor meBelmont.pdf

Compas, B. E., Forehand, R., Thigpen, J., Hardcastle, E., Garai, E., McKee, L.,...Bettis, A. (2015). Efficacy and moderators of a family group cognitive-behavioral preventive intervention for children of parents with depression. Journal of Consulting and Clinical Pychology, 83(3), 541-553. 
doi:org/10.1037/a0039053

Daset, L. R., López, C., \& Hidalgo, M. D. (2009). Síndromes empíricos en una población adolescente evaluados mediante el YSR. Ciencias Psicológicas, 3(1), 67-82.

Deighton, J., Croudace, T., Fonagy, P., Brown, J., Patalay, P., \& Wolpert, M. (2014). Measuring mental health and wellbeing outcomes for children and adolescents to inform practice and policy: A review of child self-report measures. Child Adolescent Psychiatry Mental Health, 8(1), 1-14. doi:10.1186/1753-2000-8-14

Ferrando, P., \& Anguiano, C. (2010). El análisis factorial como técnica de investigación en psicología. Papeles del Psicólogo, 31(1), 1833

Freire, T., Teixeira, A., Silva, E., \& Matias, G. P. (2014). Interventions for youth depression: From symptom reduction to well-being and optimal functioning. Journal of Behavior, Health \& Social Issues, 6(2), 9-19. doi:10.5460/jbhsi.v6.2.50816

García, M., Rivera, S., Reyes, I., \& Díaz-Loving, R. (2006). Construcción de una escala de funcionamiento familiar. Revista Iberoamericana de Diagnóstico y Evaluación - e Avaliação Psicológica, 2(22), 91-110.

Ivanova, M., Achenbach, T.M., Rescorla, L., Dumenci, L., Almqvist, F., Bilenberg, N.,...Zukauskiene, R. (2007). The generalizability of the Youth Self-Report syndrome structure in 23 societies. Journal of Consulting and Clinical Psychology, 75(5), 729-738. doi:10.1037/0022-006X.75.5.729

Kieling, C., Baker-Henningham, H., Belfer, M., Conti, G., Ertem, I., Omigbodun, O.,...Rahman, A. (2011). Child and adolescent mental health worldwide: evidence for action. The Lancet, 378(9801), 1515-1525.

Kline, T. (2005). Psychological testing: A practical approach to design and evaluation. New York: Sage Publisher.

Lacalle, M. (2009). Escalas DSM del CBCL y YSR en niños y adolescentes que acuden a consulta en servicios de salud mental. (Tesis inédita de Doctorado). Facultad de Psicología, Universidad Autónoma de Barcelona, Bellaterra.

Lemos, S., Fidalgo, A. M., Calvo, P., \& Menéndez, P. (1992a). Validación de la escala de psicopatología infanto-juvenil YSR. Clínica y Salud, (3), 183-194.

Lemos, S. Fidalgo, A. M., Calvo, P., \& Menéndez, P. (1992b). Salud mental de los adolescentes asturianos. Psicotema, 4(1), 2148.

Lemos, S., Vallejo, G., \& Sandoval, M. (2002). Estructura factorial del Youth Self-Report (YSR). Psicothema, 14(4), 816-822.

Lloret, S. Ferreres, A., Hernández, A., \& Tomás, M. (2014). El análisis factorial exploratorio de los ítems: una guía práctica, revisada y actualizada. Anales de Psicología, 30(3), 1151-1169. doi:.org/10.6018/analesps.30.3.199361

Nunnally, J. C., \& Bernstein, I. H. ( 1995). Teoría psicométrica (3a ed.). Ciudad de México: McGraw-Hill Latinoamericana.

Rescorla, L., Achenbach, T. M., Ivanova, M., Dumenci, L., Almqvist, F., Bilenberg, N.,... Verhulst, F. (2007). Epidemiological comparisons of problems and positive qualities reported by adolescents in 24 countries. Journal Consult Clinic Psychology, 75(2), 351-358. doi:10.1037/0022-006X.75.2.351

Rescorla, L., Ivanova, M., Achenbach, T. M., Begovac, I., Chahed, M., Drugli, M.,...Zhang, E. Y. (2012). International epidemiology of child and adolescent psychopathology II: Integration and applications of dimensional findings from 44 societies. Journal of the American Academic Child \& Adolescent Psychiatry, 51(12), 1273-1283.

doi:10.1016/j.jaac.2012.09.012

Roque, M. P., Acle, G., \& García, M. (2009). Escala de resiliencia materna: Un estudio de validación en una muestra de madres con niños especiales. Revista Iberoamericana de Diagnóstico y Evaluación - e Avaliação Psicológica, 1(27), 107-132.

Sandoval, M., Lemos, S., \& Vallejo, G. (2006). Self-reported competences and problems in Spanish adolescents: A normative study of the YSR. Psicothema, 18(4), 804-809.

Ullman, J. (2006). Structural equation modeling: Reviewing the basic and moving forward. Journal of Personality Assessment, 87(1), 3550.

Valencia, M., \& Andrade, P. (2005). Validez del Yough Self Report para problemas de conducta en niños mexicanos. International 
Journal of Clinical and Health Psychology, 5(3), 499-520.

Verhulst, F. \& Achenbach, T. (1995). Empirically based assessment and taxonomy of psychopathology. Cross-cultural applications. A review. European Child \& Adolescent Psychiatry, 4(2), 61-76. doi:10.1007/BF01977734

Widakowich, C. (2012). El enfoque dimensional vs el enfoque categórico en psiquiatría: Aspectos históricos y epistemólogicos. Revista Argentina de Clínica Neuropsiquiátrica, 17(4), 365-374.

Zubeidat, I., Fernández, P. A., Ortega, J. F., Vallejo, M. A., \& Sierra, J.C. (2009). Características psicosociales y psicopatológicas en una muestra de adolescentes españoles a partir del Youth Self-Report/11-18. Anales de Psicología, 25(1), 60-69. 